Revista de la Escuela de Ciencias de la Educación. 2022, Año 18 1(17), 189 204. Enero a junio. Padilla-Fuentes, G.; Rodríguez-Garcés, C. y Espinosa Valenzuela, D. Segregación y despoblamiento de la matrícula de escuelas públicas en Chile: un estudio de tendencia entre los años 2003 y 2018.

\title{
SEGREGACIÓN Y DESPOBLAMIENTO DE LA MATRÍCULA DE ESCUELAS PÚBLICAS EN CHILE: \\ UN ESTUDIO DE TENDENCIA ENTRE LOS AÑOS 2003 Y 2018
}

\author{
SEGREGATION AND DEPOPULATION OF PUBLIC SCHOOL ENROLLMENT IN \\ CHILE: A TREND STUDY BETWEEN 2003 AND 2018
}

\section{Geraldo Padilla-Fuentes \\ Universidad del Bío-Bío, Chile gpadilla@ubiobio.cl}

\section{(iD)}

\section{Carlos Rodríguez-Garcés \\ Universidad del Bío-Bío, Chile carlosro@ubiobio.cl}

\section{(iD)}

\section{Denisse Espinosa-Valenzuela} Universidad del Bío-Bío, Chile daespinosa@ubiobio.cl

Recibido: 25 de junio de 2021 Aprobado: 28 de noviembre de 2021 Publicado: 31 de diciembre de 2021

Cita sugerida: Padilla-Fuentes, G.; Rodríguez-Garcés, C. y Espinosa Valenzuela, D. (2022).

Segregación y despoblamiento de la matrícula de escuelas públicas en Chile: un estudio de tendencia entre los años 2003 y 2018. Revista de la Escuela de Ciencias de la Educación. 1(17), 189-204.

\section{RESUMEN}

El objetivo de este artículo es analizar el comportamiento de la matrícula en los ciclos de Educación Básica y Media del sector público en Chile. Desde un enfoque cuantitativo y con técnicas descriptivas-analíticas, se exploraron datos del Ministerio de Educación de Chile y de la Encuesta de Caracterización Socioeconómica Nacional (CASEN), exhibiendo la tendencia de la matrícula y perfiles de la demanda en educación pública entre los años 2003 y 2018. Los resultados muestran un sostenido decaimiento en los niveles de matrícula municipal, especialmente desde las clases medias. Estos colegios parecen cada 
Revista de la Escuela de Ciencias de la Educación. 2022, Año 18 1(17), 189 204. Enero a junio. Padilla-Fuentes, G.; Rodríguez-Garcés, C. y Espinosa Valenzuela, D. Segregación y despoblamiento de la matrícula de escuelas públicas en Chile: un estudio de tendencia entre los años 2003 y 2018.

vez más relegados a la atención preferente de grupos vulnerables con bajo capital socioeconómico, lo cual reproduce la segregación educativa, en especial cuando se considera el crecimiento del sector privado. Las conclusiones argumentan que el deterioro evidenciado en la matrícula y prestigio social de la educación pública pueden estar llegando a un punto de no retorno donde la intervención del Estado es urgente; este debe modificar su rol subsidiario y fortalecer la misión cultural de la educación, paso indispensable para la recuperación de la convivencia y encuentro con otros en la escuela.

Palabras clave: Acceso a la educación - Educación pública - Segregación educativa - Capital social - Elección educativa.

\section{ABSTRACT}

The objective of this article is to analyze the behavior of enrollment in Basic and Secondary Education in the public sector in Chile. From a quantitative approach and with descriptive-analytical techniques, data from the Chilean Ministry of Education and the National Socioeconomic Characterization Survey (NSCS) were explored, showing the trend of enrollment and demand profiles in public education between 2003 and 2018. The results show a sustained decline in public enrollment levels, especially from the middle classes. Schools in this sector seem increasingly relegated to preferential attention to vulnerable groups with low socioeconomic capital, which reproduces educational segregation, especially when considering the growth of the private sector. The conclusions argue that the deterioration evidenced in the enrollment and social prestige of public education may be reaching a point of no return where State intervention is urgent; the State must modify its subsidiary role and strengthen the cultural mission of education, an indispensable step for the recovery of coexistence and encounter with others at school.

Keywords: Access to education - Public education - School segregation Social capital - Educational choice.

\section{INTRODUCCIÓN}

Para las naciones, la educación se configura como el mecanismo fundamental de desarrollo y crecimiento, proceso permanente de aprendizajes encargado de formar ciudadanos íntegros y autónomos. Con la intención de potenciar el logro de estos objetivos, los distintos gobiernos han intervenido la provisión, aseguramiento y calidad de sus sistemas educativos. En el caso chileno, a principios del siglo XX el Estado se caracterizaba por ser garante, proveedor y regulador del servicio educativo, buscando igualar oportunidades entre todas y todos.

Su responsabilidad y atribuciones perduraron hasta la entrada en vigencia de la reforma educativa de 1980, la cual introdujo el rol subsidiario y el principio de libre elección, favoreciendo el proceso de desarticulación y privatización de la educación pública. Se inicia así el traspaso de administración hacia los municipios 
Revista de la Escuela de Ciencias de la Educación. 2022, Año 18 1(17), 189 204. Enero a junio. Padilla-Fuentes, G.; Rodríguez-Garcés, C. y Espinosa Valenzuela, D. Segregación y despoblamiento de la matrícula de escuelas públicas en Chile: un estudio de tendencia entre los años 2003 y 2018.

y la masiva creación de instituciones escolares con gerencia privada, incentivados por la Ley Orgánica Constitucional de Enseñanza promulgada en 1990, la cual reconocía la autonomía para abrir, organizar y mantener establecimientos educacionales con mínimos requisitos (Gobierno de Chile, 1990; OCDE, 2004). Las sucesivas reformas realizadas desde la recuperación de la democracia nunca cuestionaron los principios y mecanismos que inspiraron estos cambios, más bien, las modificaciones y acciones compensatorias resultaron insustanciales, cuando no con efectos perversos.

En efecto, todas las reformas implementadas en educación después del advenimiento de la democracia mantuvieron incólume el sistema de financiamiento y el rol subsidiario del Estado en la provisión de servicios sociales como el educativo. El modelo de financiamiento de voucher inaugurado en los $80^{\prime}$, consistente en el pago de un importe o subvención estatal por alumno matriculado con independencia de la naturaleza pública o privada del establecimiento al que asista, es considerado en la actualidad como la piedra de tope para una transformación estructural del sistema.

Sobre la base de la reforma educativa del ' 80 y las enmiendas realizadas con posterioridad a la recuperación de la democracia en Chile, el sistema educativo en su componente administrativo se configuró en cuatro tipologías: colegios públicos de administración municipal, colegios particulares subvencionados, establecimientos particulares pagados $y$, aunque con una presencia más marginal en la oferta educativa, estarían los colegios de administración delegada, instituciones de propiedad estatal cuya gestión administrativa ha sido transferida a corporaciones de carácter privado. Todas estas instituciones, con excepción de los colegios particulares pagados, reciben financiamiento estatal, aunque los establecimientos particulares subvencionados tendrían la posibilidad de cobrar un importe adicional a las familias.

Paralelamente en 1988, y recogiendo la experiencia acumulada los últimos 20 años por los sistemas nacionales de evaluación de resultados de aprendizaje, se creó el Sistema de Medición de la Calidad de la Educación (SIMCE), mecanismo que mediante la aplicación de pruebas estandarizadas busca proveer de información a los distintos actores del sistema para realizar ajustes curriculares pertinentes, promover la competencia entre establecimientos y orientar la elección educativa de padres, madres y apoderados.

Por otra parte, la modificación en 1994 a la Ley de Subvenciones Educacionales del Estado, donde se aprobó el financiamiento compartido (copago) entre el Estado chileno y la familia, sostenía que, a mayor oferta y competencia, mayor calidad educativa. Esto bajo el supuesto que las elecciones parentales se efectúan sobre la base de criterios de eficiencia en sintonía con el Proyecto Educativo de cada institución. Se estimaba que el copago posibilitaría aumentar la inversión por alumno a través del aporte realizado por padres y madres; inversión parental que incrementaría los vínculos y nivel de compromiso con la escuela.

La relación virtuosa atribuida al aporte económico familiar como eficiente mecanismo para allegar recursos y fortalecer el compromiso parental con la labor 
Revista de la Escuela de Ciencias de la Educación. 2022, Año 18 1(17), 189 204. Enero a junio. Padilla-Fuentes, G.; Rodríguez-Garcés, C. y Espinosa Valenzuela, D. Segregación y despoblamiento de la matrícula de escuelas públicas en Chile: un estudio de tendencia entre los años 2003 y 2018.

educativa, rápidamente tornó a consecuencias indeseadas (Falabella, 2015). Entre sus efectos más notorios e inmediatos está el aumento significativo de la oferta privada, la mercantilización educativa y la profundización de los procesos de segmentación. En efecto, la desregulación del sistema, su funcionamiento con lógicas de mercado y los incentivos instalados mediante subvención y copago, propiciaron la creación y ampliación de instituciones de carácter privado con fines de lucro, los cuales bajos distintos mecanismos y estrategias captan cada vez mayor matrícula (Santos y Elacqua, 2016).

La oferta privada se hace masiva, segmentada y diversa con la finalidad de atender a un alumnado heterogéneo, transformando las estructuras del sistema educativo. Tras la lógica de libre elección, a la que suele atribuirse una función intrínseca y fundamental para la calidad y eficiencia educativa (Hernández y Raczynski, 2015), se esconde la configuración de distintas tipologías de colegio para distintos grupos de estudiantes que no han hecho más que segmentar a las familias según su capacidad de pago y capital sociocultural, replicando la pirámide social y segregación residencial en el espacio educativo (Mizala y Torche, 2012; Elacqua y Santos, 2013).

Aunque la clásica adscripción de estudiantes de clases altas a colegios pagados, clases medias a particulares subvencionados y clases bajas a públicos resulta parcialmente anacrónica con la irrupción del copago, el vínculo entre clase social y elección educativa se refina y persiste. En este contexto, la extensión de la oferta particular subvencionada ha propiciado su mixturización interna, atendiendo ahora a estudiantes de diferentes estratos socioeconómicos, pero siempre en un espacio de segmentación institucional (OCDE, 2011; Flores y Carrasco, 2013; Córdoba et. al., 2016). Así entendido, los establecimientos particulares subvencionados pueden ser desagregados en diversas tipologías sobre la base de los atributos socioeducativos de su alumnado y/o el perfil del sostenedor. Así por ejemplo, el estudio de (González y Parra, 2016) reporta que es posible caracterizar a este sector educativo también a partir de las redes económicas, ideológicas y políticas que los sostenedores ostentan.

Por su parte, la escuela pública ha enfrentado un progresivo deterioro en su infraestructura y prestigio social, atendiendo preferentemente a los segmentos de menores ingresos (García-Huidobro, 2010; Gubbins, 2013; Bellei, 2018), aunque ello no significa necesariamente que este último se encuentre satisfecho con la elección que ha realizado (Hernández y Raczynski, 2015). En el cumplimiento de su misión, estas instituciones enfrentan una serie de problemáticas personales y familiares de sus estudiantes, a lo que se suma el limitado apoyo educativo que reciben desde los hogares más vulnerables donde usualmente los niveles de escolaridad son bajos y los recursos para adquirir materiales o complementos de estudio escasean (Bellei, 2015).

Con la persistencia del rol subsidiario del Estado en materia educativa, desde los ' 90 ha crecido la irrupción de escuelas pertenecientes al sector privado en el sistema, todo lo cual, por una parte, ha perjudicado los niveles de matrícula pública y su atractivo entre las familias, y por otra, ha permitido el enquistamiento de las lógicas de competencia en la provisión de este servicio social. En este 
Revista de la Escuela de Ciencias de la Educación. 2022, Año 18 1(17), 189 204. Enero a junio. Padilla-Fuentes, G.; Rodríguez-Garcés, C. y Espinosa Valenzuela, D. Segregación y despoblamiento de la matrícula de escuelas públicas en Chile: un estudio de tendencia entre los años 2003 y 2018.

contexto, la libre elección parental y selección escolar institucional funcionaron como mecanismos reproductores de desigualdad y segregación sociocultural entre los sectores públicos y privados, separando a las familias en razón de sus características socioeducativas o prerrogativas de clase (Cucchiara, 2013; Cal et. al., 2019).

En teoría, el sistema educacional reconoce el principio de libre elección para que padres y madres escojan, con independencia de su lugar de residencia y de acuerdo con sus preferencias e intereses, el establecimiento educativo de sus hijos/as. Sin embargo, también permitía a los colegios aplicar mecanismos de selección en sus procesos de admisión sobre la base de requisitos académicos del estudiante o exigencias a las familias. Esta práctica institucional restringe profundamente el principio de libre elección y favorece la segregación y segmentación socioeducativa, además que obstaculiza la convivencia y encuentro con otros en la escuela (Bellei, 2007; Valenzuela, 2008; Contreras et. al., 2010).

Dentro del panorama decisional de padres y madres de mayor capital económico y educativo, la oferta privada se configura como la opción más elegible y adecuada para la educación de sus hijos/as, con apropiados atributos de seguridad, calidad y/o eficiencia (Hernández y Raczynski, 2015). Por otro lado, desde su perspectiva la educación pública presenta dificultades para transitar por el currículum, problemas de convivencia escolar, déficit de infraestructura y equipamiento, además de no garantizar el ingreso a la educación superior (Departamento de Educación Pública, 2018); atributos que la convertirían en un lugar de riesgo en vez de oportunidad para el contacto y convivencia (Tijmes, 2012).

Así, las elecciones parentales que rehúyen de la oferta pública serían reflejo de una insatisfacción de expectativas acerca de la calidad y eficiencia educativa, además del esfuerzo por sostener status y proveer seguridad a los hijos/as en colegios de alta homogeneidad sociocultural. En este sentido, Van Zanten (2003) postula que las elecciones educativas de padres y madres de clase media se encuentran vinculadas con la valoración de características culturales y de convivencia de la escuela; criterios que favorecen al sector privado en lugar del público, por cuanto se le percibe menos expuesto a violencia, problemas de convivencia y garantizan un mejor aprovechamiento educativo.

En atención al panorama de cuestionamiento y deterioro de la educación pública reportados, el objetivo de este artículo es analizar las fluctuaciones que han experimentado las escuelas públicas en su matrícula entre los años 2003 y 2018, indagando en los atributos socioeconómicos de quienes la escogen y los procesos de segmentación socioeducativa que se instalan y profundizan en un modelo de financiamiento que subsidia la demanda. Para esto, se utilizan los registros del Ministerio de Educación de Chile e información de la Encuesta de Caracterización Socioeconómica Nacional (CASEN), calculando proporciones e índices que reflejen, por un lado, el despoblamiento de la educación pública y, por otro, el perfil de quienes la eligen. 
Revista de la Escuela de Ciencias de la Educación. 2022, Año 18 1(17), 189 204. Enero a junio. Padilla-Fuentes, G.; Rodríguez-Garcés, C. y Espinosa Valenzuela, D. Segregación y despoblamiento de la matrícula de escuelas públicas en Chile: un estudio de tendencia entre los años 2003 y 2018.

\section{DESARROLLO}

\section{Metodología}

\section{Diseño}

Desde una perspectiva cuantitativa, este artículo analiza la tendencia de la matrícula que han reportado los establecimientos públicos en Chile para sus ciclos de Enseñanza Básica y Media entre los años 2003 y 2018. A través de indicadores desagregados de cobertura escolar, se indaga sobre la merma en el volumen de matrículas de este sector, además, se utilizan algunos atributos económicos y socioculturales de la demanda para establecer perfiles de adhesión.

\section{Instrumentos}

Para el análisis estadístico se utilizaron dos fuentes de datos nacionales. Por medio de la Ley 20285 sobre acceso y transparencia a la información, se tuvo acceso al registro histórico de matrículas del Ministerio de Educación (Mineduc). Estas bases de datos contienen información sobre matrícula, oferta educativa y niveles de enseñanza de cada unidad escolar, organizadas por año y tipo de administración. Información que fue condensada y analizada según los requerimientos del estudio.

La segunda fuente de datos corresponde a la Encuesta de Caracterización Socioeconómica Nacional (CASEN) del año 2017, principal herramienta para la evaluación de políticas públicas y diagnóstico socioeconómico en Chile. Esta encuesta es de aplicación bianual y recoge información sobre salud, trabajo, educación, ingresos y condiciones de vida de las personas, con énfasis en la vulnerabilidad de los grupos más pobres. Cabe mencionar que se trabaja con CASEN 2017 porque el acceso a la versión 2019 se ha retrasado en razón de la pandemia por COVID-19 y la crisis económica y sanitaria que ha ocasionado. De hecho, el Ministerio de Desarrollo Social y Familia, entidad encargada de CASEN, ha preparado una versión especial de la encuesta (CASEN en Pandemia 2020), limitando la disposición de datos hasta nuevo aviso.

\section{Muestra}

Los datos de matrícula están agrupados según establecimiento y ciclo educativo. Para el año 2018, Mineduc cuenta con 16.044 colegios registrados (Tabla 1), de los cuales solo un $42,4 \%$ corresponde a dependencias Municipales o públicas. Coincidente con los movimientos de urbanización del país, la mayoría de colegios opera en zonas urbanas $(92,0 \%)$, mientras que un $72,5 \%$ atiende preferentemente a población vulnerable o de estratos socioeconómicos Bajo y Medio Bajo, que, en la tipificación utilizada por el Mineduc en razón del capital económico y educativo parental, corresponde a las categorías A y B. 
Revista de la Escuela de Ciencias de la Educación. 2022, Año 18 1(17), 189 204. Enero a junio. Padilla-Fuentes, G.; Rodríguez-Garcés, C. y Espinosa Valenzuela, D. Segregación y despoblamiento de la matrícula de escuelas públicas en Chile: un estudio de tendencia entre los años 2003 y 2018.

Tabla 1: descripción de la muestra Mineduc 2018 (\%)

\begin{tabular}{|l|c|c|}
\hline & $\begin{array}{c}\text { Colegios } \\
(\mathrm{N}=16.044)\end{array}$ & $\begin{array}{c}\text { Matrículas } \\
(\mathrm{n}=2.885 .517)\end{array}$ \\
\hline Dependencia & & \\
- Municipal & 42,4 & 37,3 \\
- Particular subvencionado & 47,4 & 53,3 \\
- Particular privado & 10,2 & 9,4 \\
\hline Zona & 69,2 & 92,0 \\
- Urbana & 30,8 & 8,0 \\
- Rural & & \\
\hline GSE & 72,5 & 56,1 \\
- A y B & 17,3 & 25,8 \\
- C & 10,2 & 18,1 \\
- D y E & & \\
\hline Ciclo & - & 68,9 \\
- Enseñanza Básica & - & 31,1 \\
- Enseñanza Media & & \\
\hline
\end{tabular}

Nota: $a=$ no todos los colegios cuentan con su clasificación socioeconómica, por lo que se incluyen solo aquellos etiquetados $(\mathrm{N}=8.445)$.

Elaboración propia con datos Mineduc (2018).

En 2018, estos colegios atendieron a un total de 3.582.448 estudiantes en distintos ciclos, niveles y regímenes. De este universo, fueron seleccionadas aquellas matrículas que pertenecieran a la modalidad regular de estudios y en los ciclos de Enseñanza Básica y Media, ya fuera Técnica o Humanista. De este modo, la muestra estuvo constituida por 2.885.517 matrículas. Al momento de observar cómo se distribuyen los estudiantes, encontramos que alrededor de 2 de cada 5 asisten a educación pública $(37,3 \%)$, mayoritariamente a colegios vulnerables $(56,1 \%)$.

Los perfiles de la demanda educativa del sector público se configuraron sobre la base de los datos reportados por la encuesta CASEN 2017, cuya muestra la integran 21.866 estudiantes.

\section{Procedimiento}

Una vez revisadas las bases de datos y habiendo controlado sesgos de información como datos perdidos o códigos de respuesta inválidos, se procedió a seleccionar la muestra principal de estudios, constituida por las matrículas de los establecimientos adscritos al sistema público y con modalidades regulares de Enseñanza Básica y Media. De este modo, la Figura 1 presenta la evolución que han tenido estos registros desde el año 2004 al 2018 en dos sentidos: cobertura y pérdida de volumen.

Luego se analizan las fluctuaciones en la matrícula en la educación pública según ciclo de estudios y características socioeconómicas de la demanda. Cabe destacar que, a diferencia del Mineduc, CASEN sí reporta datos para el año 2003, lo cual permite ampliar levemente la franja temporal de estudio. Junto con los niveles de captación de matrículas, interesa ver cómo se manifiesta el despoblamiento entre los extremos temporales y por cada año comprendido en 
Revista de la Escuela de Ciencias de la Educación. 2022, Año 18 1(17), 189 204. Enero a junio. Padilla-Fuentes, G.; Rodríguez-Garcés, C. y Espinosa Valenzuela, D. Segregación y despoblamiento de la matrícula de escuelas públicas en Chile: un estudio de tendencia entre los años 2003 y 2018.

los registros. Para tal efecto, se elaboran los índices de diferencia relativa [ $\mathrm{Vt}_{0}{ }^{-}$ $\left.\mathrm{V} \mathrm{t}_{n} / \mathrm{Vt}_{n}\right]$ y la tasa de crecimiento anual compuesto $\left[\left(\left(\mathrm{Vt}_{\mathrm{n}} / \mathrm{Vt}_{0}\right)^{\wedge} 1 / \mathrm{t}_{\mathrm{n}}-\mathrm{t}_{0}\right)-1\right]$.

\section{Resultados y discusión}

Al año 2018, un total de 2.885.517 niños, niñas y adolescentes participan del sistema escolar chileno, alcanzando coberturas netas del orden del $93 \%$ en el ciclo de enseñanza básica y $87 \%$ para enseñanza media. Si bien estas cifras en los niveles de escolarización significan un progreso importante, autores como Brunner y Elacqua (2003) y Bellei (2018) afirman que su desarrollo histórico ha sido lento, tardío y desigual, bajo un esquema de profunda segmentación socioeducativa. Aun cuando la diferenciación en el acceso y calidad de la educación recibida ha sido un problema estructural, la reforma de 1980 acentuó las brechas instalando profundos cambios en la forma en que se comprende y provee el servicio educativo en el país.

En efecto, la matrícula del sector público arrastra una merma constante desde la década de los $90^{\prime}$, cubriendo al año 2018 un escaso 32,4\% y 38,2\% de los ciclos de Educación Media y Básica respectivamente (Figura 1). Esta disminución representa una pérdida bruta del $30 \%$ en comparación a la registrada el año 2004. Mientras la cantidad de matriculados en educación pública decae, el sector privado, en especial el segmento particular subvencionado, acrecienta su volumen de matrícula, atendiendo a cerca de dos tercios $(63,4 \%)$ de la población estudiantil. Volúmenes de participación privada que, a juicio de autores como Bellei (2018), situaría a Chile como uno de los sistemas educativos más privatizados del mundo.

Figura 1. Cobertura y pérdida de matrículas en Educación Municipal según ciclos de enseñanza

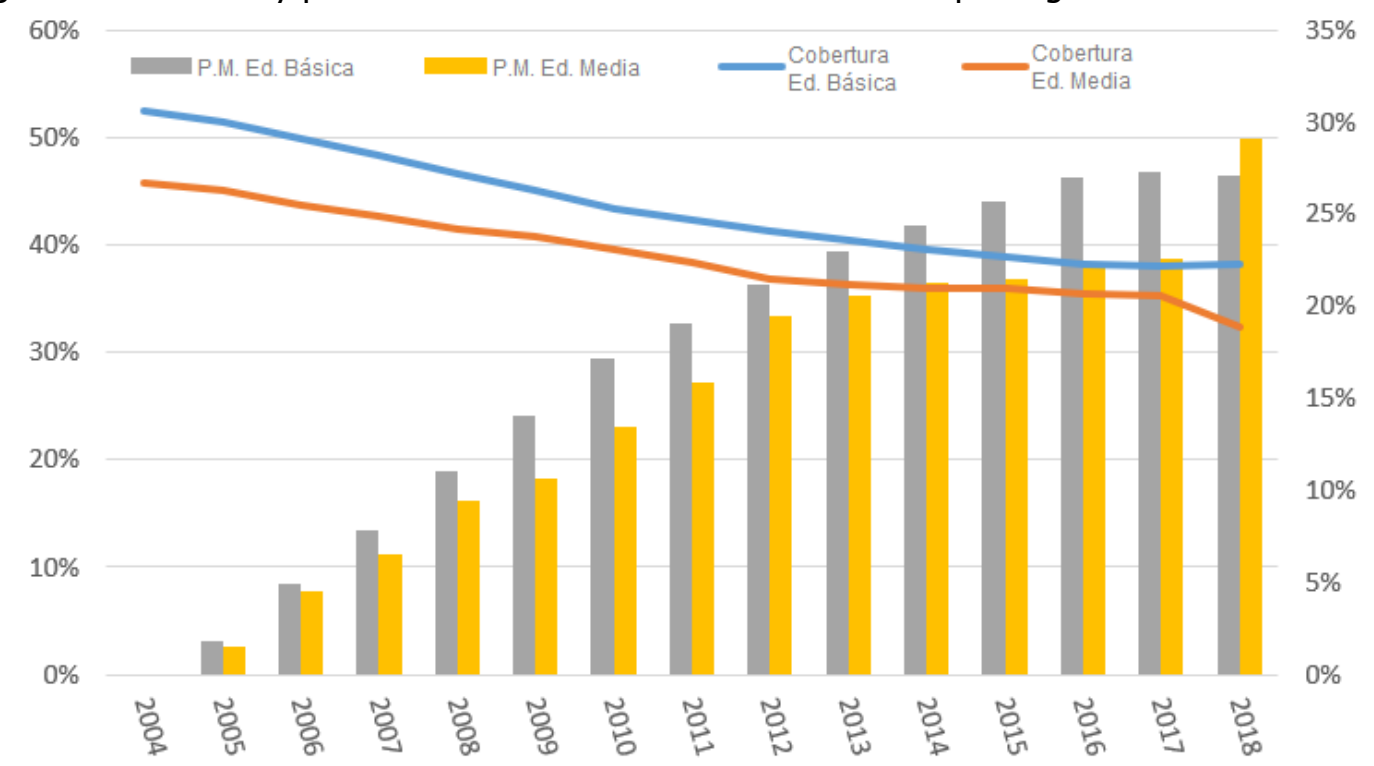

Nota: P.M= Pérdida de matrícula en puntos porcentuales; Ed.= Ciclo educativo. Elaboración propia con datos Mineduc, años respectivos.

Uno de los factores que comúnmente la literatura vincula con este 
Revista de la Escuela de Ciencias de la Educación. 2022, Año 18 1(17), 189 204. Enero a junio. Padilla-Fuentes, G.; Rodríguez-Garcés, C. y Espinosa Valenzuela, D. Segregación y despoblamiento de la matrícula de escuelas públicas en Chile: un estudio de tendencia entre los años 2003 y 2018.

despoblamiento de la educación pública es el rendimiento insuficiente que obtiene en los procesos de medición de calidad educativa. Tanto el SIMCE como pruebas estandarizadas internacionales han dado cuenta del retraso curricular, dificultades de aprendizaje y bajo rendimiento que tienen las instituciones públicas en comparación a las privadas, a lo que se suma un déficit en sus condiciones de seguridad, respeto y organización (Contreras et al., 2007; Lomas, 2007; López et al., 2011; Tijmes, 2012; Agencia de Calidad de la Educación, 2017); conjunto de elementos que tienden a ser considerados en las decisiones parentales educativas.

Asimismo, investigaciones plantean que la falta de restricciones en la admisión pública configuraría contextos expuestos al roce con estudiantes vulnerables, lo cual para padres y madres de clases medias y altas pondría en riesgo el capital social y cultural de la familia (Forsey et al., 2008; Rich y Jennings, 2015). En la medida que el financiamiento compartido, la selección escolar y las prácticas de competencia favorecen la gestión de los colegios privados, se produce un cierre socia/llamativo para padres y madres que evalúan la educación como una inversión a proteger, abandonando paulatinamente el sector público (Hernández y Raczynski, 2015).

Por lo anterior, el despoblamiento de la educación pública observado en la Figura 1 sería en gran medida un fenómeno migratorio hacia la oferta privada, especialmente la de tipo particular subvencionada, ya sea en búsqueda de mejores resultados escolares, reproducción de estatus o espacios de convivencia más seguros.

En este contexto, lo que autores como García-Huidobro (2010) han denominado crisis de la educación pública, convierte a este sector en opción de una demanda específica (Quijada y Mungarro, 2014). Están los que eligen la educación pública en tanto proveedora de un servicio gratuito y de calidad; quienes por motivos prácticos y a fin de no interrumpir trayectorias escolares 0 vínculos relacionales prefieren no migrar y, en peor posición, están quienes la escogen por la imposibilidad de acceder a otra oferta. En este último grupo se aplica lo que hemos denominado política de elección educativa por única opción, es decir, cuando la elección de colegios públicos no refleja predilección, gusto o interés hacia el proyecto educativo, sino que constituye la única opción asequible; circunscrita mayoritariamente a población vulnerable.

Al respecto, la Tabla 2 informa sobre la variación de matrícula en el sector público según atributos socioeducativos de su demanda. La escuela pública se ha configurado como opción preferente de población socioeconómicamente vulnerable y de bajo capital familiar educativo. Al año 2018, alrededor de 3 de cada $5(61,6 \%)$ estudiantes pertenecientes a familias ubicadas en el decil 1 asisten a colegios Municipales en educación Básica, mientras que en Media lo hace el $56,7 \%$. Por su parte, quienes provienen de familias clasificadas en el decil 10 , registran tan solo un $7,7 \%$ y $9,4 \%$ de participación en cada ciclo respectivamente. Si bien en ambos niveles educativos las pérdidas relativas de matrícula son similares entre los periodos analizados (en torno al 15\%), cabe destacar que la baja participación de clases altas en las escuelas públicas ha sido 
Revista de la Escuela de Ciencias de la Educación. 2022, Año 18 1(17), 189 204. Enero a junio. Padilla-Fuentes, G.; Rodríguez-Garcés, C. y Espinosa Valenzuela, D. Segregación y despoblamiento de la matrícula de escuelas públicas en Chile: un estudio de tendencia entre los años 2003 y 2018.

una constante histórica, particularmente acentuada tras la reforma del '80.

Tabla 2. Variación de matrícula en el sector público según atributos socioeducativos de la demanda

\begin{tabular}{|l|c|c|c|c|c|c|c|c|}
\cline { 2 - 8 } & \multicolumn{9}{c|}{ Captación } & Diferencia relativa & \multicolumn{2}{c|}{ TCAC } \\
\cline { 2 - 8 } & 2003 & 2018 & $2003-2018$ & $2003-2018$ \\
\cline { 2 - 8 } & Básica & Media & Básica & Media & Básica & Media & Básica & Media \\
\hline Deciles de ingreso \\
\hline - Decil 1 & 72,8 & 67,4 & 61,6 & 56,7 & $-15,4$ & $-15,9$ & $-1,2$ & $-1,2$ \\
\hline - Decil 10 & 8,5 & 11,1 & 7,7 & 9,4 & $-9,4$ & $-15,3$ & $-0,7$ & $-1,2$ \\
\hline Jefe/a de núcleo con Estudios Superiores \\
\hline - No & 64,1 & 57,8 & 55,4 & 52,6 & $-13,6$ & $-9,0$ & $-1,0$ & $-0,7$ \\
\hline$-\mathrm{Si}$ & 39,5 & 40,2 & 26,7 & 26,3 & $-32,4$ & $-34,6$ & $-2,8$ & $-3,0$ \\
\hline
\end{tabular}

Nota: TCAC $=$ Tasa de crecimiento anual compuesto, medida que controla la fluctuación de valores entre unidades de tiempo.

Elaboración propia con resultados Encuesta de Caracterización Socioeconómica Nacional (CASEN) de los años 2003 y 2018.

La ausencia de mecanismos de selección como atributo predominante de la escuela pública haría de esta una opción menos deseable para padres y madres de sectores con ingresos medios y altos. Para estos actores, la admisión sin restricción por perfil académico o familiar del estudiante pondría en riesgo la convivencia, disciplina y calidad de la enseñanza impartida, dificultando la labor educativa en las aulas. Tal como han recogido Hernández y Raczynski (2015), los padres y madres estiman que en contextos educativos mixturados socialmente deben desviarse recursos desde lo pedagógico hacia la solución de problemas personales y/o familiares de los estudiantes. Aun cuando impresiones como estas se encuentran fuertemente influenciadas por el prejuicio, existe evidencia sobre las dificultades que entraña la labor educativa en contextos pobres o de carencia, sobre todo respecto a convivencia, vinculación familia-escuela y condiciones de educabilidad en la sala de clases (Castro y Cano, 2013).

Por tanto, la elección educativa parental es correlato de la disponibilidad de recursos económicos y educativos del grupo familiar, del conjunto de expectativas que definen la conservación/reproducción de estatus en la familia y del grado de aislamiento que se instala respecto a los estudiantes indeseados. Esto añade al análisis de la segregación escolar propiciada por la subsidiariedad del Estado en educación, la aspiración de padres y madres por ingresar a los colegios perimetrados socialmente, evitando así a quienes se evalúa como conflictivos, de lento aprendizaje y con problemas familiares; lo que constituye al despoblamiento de la educación pública en un fenómeno de clases.

La dinámica de distinción social por nivel socioeconómico es reforzada por mecanismos de acumulación de capital cultural o educativo. De este modo, los padres y madres que alcanzan estudios superiores optarían mayoritariamente por la oferta privada, elección que se ha masificado los últimos años. Para el caso de 
Revista de la Escuela de Ciencias de la Educación. 2022, Año 18 1(17), 189 204. Enero a junio. Padilla-Fuentes, G.; Rodríguez-Garcés, C. y Espinosa Valenzuela, D. Segregación y despoblamiento de la matrícula de escuelas públicas en Chile: un estudio de tendencia entre los años 2003 y 2018.

enseñanza Media, en el año 2003 un 40,2\% de los padres y madres con estudios superiores optaba por educación pública, mientras que para el año 2018 la cifra se reduce un tercio (Tabla 2 ).

El capital humano acumulado por padres y madres correlaciona no solo con el nivel de ingresos familiar, sino también con las expectativas intergeneracionales. De allí que la inversión educativa realizada por estas familias no pondere tan solo aspiraciones académicas, sino también las oportunidades para la articulación de redes y movilidad social que, a su criterio, ofrecería en mayor magnitud y calidad la oferta privada. El sistema educativo posibilita, cuando no favorece, la articulación de estos mecanismos de distinción y cierre social con lógicas de inclusión/exclusión basadas en el capital socioeconómico y educativo, estableciendo así una alta homogeneidad al interior de las comunidades a la vez que maximiza la varianza entre ellas.

Si bien la escuela pública constituye un espacio de atención preferente a sectores socioeconómicos bajos y medio-bajos, alumnos pertenecientes a estos segmentos también migran a instituciones de administración privada, aunque la pérdida de matrícula es menos acentuada, particularmente cuando la familia dispone de bajo capital educativo acumulado. La continua ampliación de la oferta privada, el financiamiento compartido y la atracción de quienes poseen mejores perfiles académicos, unido a componentes aspiracionales de las familias, favorecen el desplazamiento desde lo público hacia un determinado segmento de la oferta privada. En efecto, al año 2018 alrededor de 2 de cada 5 estudiantes del $10 \%$ más pobre estudia en colegios particulares subvencionados por el Estado, los cuales a juicio de la demanda destacan por atributos simbólicos y prácticos en un contexto donde la oferta pública está fuertemente restringida.

En suma, el despoblamiento de la educación pública no significa tan solo una migración o movilidad educativa desde los sectores municipales hacia los particulares, pues esto puede ser explicado también teniendo en cuenta la fuerte irrupción de oferentes privados y la serie de incentivos que la política educativa estableció para la atracción y retención de estudiantes en este sector (Paredes y Pinto, 2009). En consecuencia, se hace necesario incluir al análisis el contexto en que se sitúa el quehacer educativo de los establecimientos públicos, la desigualdad de condiciones con que opera y el tipo de problemáticas que afronta previo y durante el tránsito curricular.

\section{CONCLUSIÓN}

La educación pública en Chile ha estado expuesta a un panorama árido y desventajoso por más de tres décadas. La subsidiariedad ofrecida a los intereses privados en educación bajo el supuesto de aportar a lo público, amenazó directamente el proyecto de los establecimientos estatales. La irrupción y masificación de colegios particulares no tan solo redefinieron el papel del Estado en materia educativa, sino también las condiciones, exigencias y limitantes con que operaría la escuela pública. Si bien las actuales reformas educativas han comprometido un nuevo trato hacia este sector, persiste una legítima 
Revista de la Escuela de Ciencias de la Educación. 2022, Año 18 1(17), 189 204. Enero a junio. Padilla-Fuentes, G.; Rodríguez-Garcés, C. y Espinosa Valenzuela, D. Segregación y despoblamiento de la matrícula de escuelas públicas en Chile: un estudio de tendencia entre los años 2003 y 2018.

incertidumbre respecto de su real capacidad para lograr las mejoras anheladas.

En tal sentido, los resultados de este artículo definen las trayectorias y perfiles que registra la adscripción a la escuela pública. Los resultados develan una progresiva y preocupante pérdida en la captación de matrícula, situación que pone en riesgo la continuidad operativa de muchas instituciones educativas bajo el actual modelo de financiamiento. El refinamiento de los perfiles académicos y socioculturales del alumnado a través de mecanismos de selección y copago han sido estrategias ampliamente utilizadas por la oferta privada para mejorar su posicionamiento, por cuanto sus resultados estarían más vinculados al control de admisión que a atributos propios de este tipo de administración.

En este panorama de competencia desleal, la oferta pública se restringe y se le atribuye escasa eficiencia y eficacia educativa. A esto debemos añadir la nueva postura que asumen padres y madres respecto al valor de la educación, entendida como inversión tendiente a la conservación y reproducción de estatus, donde la escuela pública aparece como escasamente deseable. En efecto, significativos conglomerados poblacionales rehuyen de la escuela pública optando por la oferta particular subvencionada incluso con relativa independencia de los niveles de logro que estas alcancen. En una dinámica de distinción y agrupamiento según Canales, Bellei y Orellana (2016) y Hernández y Raczynski (2015), los padres y apoderados buscan separarse de lo que consideran la turba o los indeseables como resultado de una socio-fobia, mientras paralelamente desarrollan acciones de socio-filia o concertación con aquellos que conciben como sus iguales o aspiracionalmente deseables.

De allí que padres, madres y apoderados distingan, en una lógica tan aspiracional como arribista, entre aquellos colegios que "seleccionan" y aquellos que "mezclan" desarrollando, sobre la base de sus decisiones parentales educativas, mecanismos de distinción y separación que proporcionando una sensación de pseuda-seguridad no hacen más que profundizar peligrosamente la estratificación socioeducativa que, como característica estructural, permea a todo el sistema educativo en sus distintos niveles.

Si bien se observa una mejora sustantiva en las tasas de cobertura del sistema educativo, ha sido a costa de su privatización y permitir la segregación entre unidades educativas. Teniendo como referencia el año 2004, en 2018 la matrícula pública ha disminuido un $30 \%$ entre los ciclos de enseñanza básica y media. Como correlato, el sector particular subvencionado ha incrementado su atractivo, siendo en la actualidad el proveedor educativo de dos tercios de la demanda.

Por el comportamiento de la cobertura pública y privada, la estructura del sistema escolar parece organizada concientemente según distinciones de clase. Se asiste a un despoblamiento del sector público que si bien es más acentuado dentro de las capas medias, también se manifiesta en los grupos más vulnerables, lo que conlleva a una mixturización de la contraparte particular subvencionada, aunque siempre en un contexto de segmentación entre escuelas. La pérdida de prestigio y la crónica falta de recursos jibariza a la escuela pública y la relega a un rol secundario y de atención preferencial a estudiantes de bajo capital cultural 
Revista de la Escuela de Ciencias de la Educación. 2022, Año 18 1(17), 189 204. Enero a junio. Padilla-Fuentes, G.; Rodríguez-Garcés, C. y Espinosa Valenzuela, D. Segregación y despoblamiento de la matrícula de escuelas públicas en Chile: un estudio de tendencia entre los años 2003 y 2018.

y educativo, profundizado por las operaciones de distinción que realizan padres y madres en sus elecciones educativas. Ello deriva en un cierre perimetral por configuración social del alumnado en espacios educativos que, por rigor, debiesen ser inclusivos.

Entre los factores analizados por la literatura consultada en lo que refiere a explicar la disminución de matrícula pública, se menciona el bajo rendimiento exhibido en comparación a la oferta privada, orientando las elecciones parentales educativas hacia instituciones, generalmente privadas, que son concebidas de mayor calidad y con adecuados climas de convivencia. Cabe hacer presente que este criterio de elección se manifestaría con menor prevalencia que los orientados a la búsqueda de status y distinción, a la vez que esta evaluación de mayor calidad de la institución, no deja de ser una suposición carente de evidencia empírica, por cuanto esta creencia parental puede incluso distar de los resultados que arrojan las pruebas estandarizadas del SIMCE una vez corregido por composición socioeconómica del alumnado. Así mismo, debido a las características del contexto en que opera la educación pública, no es de extrañar que algunos recursos deban reorientarse desde lo pedagógico hacia el apoyo personal y/o familiar de los estudiantes, cuestión que complejiza la labor educativa y afecta el desempeño curricular, reforzando su imagen social de espacio conflictivo, pauperizado y con escasos logros académicos

En conclusión, la convergencia de estos factores presiona por la puesta en marcha de un nuevo trato con la educación pública que regule pertinentemente las facultades que cada sector tiene para proveer un servicio educativo de calidad, gratuito y sin lucro, orientado al desarrollo personal y social de los y las estudiantes. La misión social que compete a la educación pública exige con urgencia un rediseño que destaque su función social, con proyectos integrales e inclusivos, lo que implica superar la noción del rol subsidiario del Estado y regular el principio de libre elección. Se precisa en consecuencia un nuevo modelo de financiamiento y revalorización de la función del Estado como garante de una educación de calidad concebida ahora como derecho para todas y todos.

\section{REFERENCIAS}

Agencia de Calidad de la Educación (2017). Resultados educativos 2017. Agencia de Calidad de la Educación.

Bellei, C. (2007). Expansión de la educación privada y mejoramiento de la educación en Chile. Evaluación a partir de la evidencia. Revista pensamiento educativo, 40(1), 1-37. https://pensamientoeducativo.uc.cl/index.php/pel/issue/view/27

Bellei, C. (2015). El gran experimento: Mercado y privatización de la educación chilena. LOM.

Bellei, C. (Ed.). (2018). La nueva educación pública. Contextos, contenidos y perspectivas de la desmunicipalización.Talleres de gráfica LOM. http://repositorio.uchile.cl/handle/2250/153153

Brunner, J. y Elacqua, G. (2003). Informe capital humano en Chile. Universidad 
Revista de la Escuela de Ciencias de la Educación. 2022, Año 18 1(17), 189 204. Enero a junio. Padilla-Fuentes, G.; Rodríguez-Garcés, C. y Espinosa Valenzuela, D. Segregación y despoblamiento de la matrícula de escuelas públicas en Chile: un estudio de tendencia entre los años 2003 y 2018.

Adolfo Ibáñez.

Cal, M.; Otero, G. y Méndez, M. (2019). Elección escolar y selección de familias: reproducción de la clase media alta en Santiago de Chile. Revista Internacional de Sociología, 77(1), e120. http://revintsociologia.revistas.csic.es/index.php/revintsociologia/article/v iew/1020

Canales, M.; Bellei, C., y Orellana, V. (2016). ¿Por qué elegir una escuela privada subvencionada? Sectores medios emergentes y elección de escuela en un sistema de mercado. Estudios pedagógicos, 42(3), 89-109. https://dx.doi.org/10.4067/S0718-07052016000400005

Castro, L. y Cano, R. (2013). Pobreza y vulnerabilidad: factores de riesgo en el proceso educativo. Contexto educativos. Revista de Educación, 16, 55-72. https://publicaciones.unirioja.es/ojs/index.php/contextos/article/view/12 90

Contreras, D.; Sepúlveda, P. \& Bustos, S. (2010). When the schools are the ones that choose: The effect of screening in Chile. Social Science Quarterly, 91(5),

$1349-1368$

http://www.econ.uchile.cl/uploads/publicacion/9abac85a9de675e45a136 e1abca14e58fcb88e4e.pdf

Contreras, M.; Corbalán, F. y Redondo, J. (2007). Cuando la suerte está hechada: estudio cuantitativo de los factores asociados al rendimiento en la PSU. REICE. Revista Iberoamericana sobre Calidad, Eficacia y Cambio en Educación, 5(5), 259-263. https://revistas.uam.es/index.php/reice/article/view/5514

Córdoba, C.; Rojas, K. y Azócar, J. (2016). Selección de alumnos y co-pago como factores de segregación escolar: Presentación de tres casos de estudio. Psicoperspectivas, 15(1), 102-116. https://www.psicoperspectivas.cl/index.php/psicoperspectivas/article/vie $\mathrm{w} / 642$

Cucchiara, M. (2013). Marketing schools, Marketing cities: who win and who loses when schools become urban amenities. University of Chicago Press. $10.7208 /$ chicago/9780226016962.001.0001

Departamento de Educación Pública. (2018). Relatos sobre la creación del Sistema Nacional de Educación Pública 2018. Dirección de Educación Pública. Ministerio de Educación. http://educacionpublica.cl/wpcontent/uploads/2018/04/Relato-sobre-la-creaci\%C3\%B3n-del-SistemaNacional-de-Educaci\%C3\%B3n-P\%C3\%BAblica-2018-4.pdf

Elacqua, G. y Santos, H. (2013). Los efectos de la elección escolar en la segregación socioeconómica en Chile: Un análisis georreferenciado. Documento de Referencia, $\mathrm{N}^{\circ} 1$. Espacio Público. https://mba.americaeconomia.com/biblioteca/papers/los-efectos-de-laeleccion-escolar-en-la-segregacion-socioeconomica-en-chile-un-an

Falabella, A. (2015). El mercado escolar en Chile y el surgimiento de la nueva gestión pública: el tejido de la política ente la dictadura neoliberal y los gobiernos de la centroizquierda (1979 a 2009). Educação \& Sociedade, 
Revista de la Escuela de Ciencias de la Educación. 2022, Año 18 1(17), 189 204. Enero a junio. Padilla-Fuentes, G.; Rodríguez-Garcés, C. y Espinosa Valenzuela, D. Segregación y despoblamiento de la matrícula de escuelas públicas en Chile: un estudio de tendencia entre los años 2003 y 2018.

36(132), 699-722. http://www.scielo.br/pdf/es/v36n132/1678-4626-es36-132-00699.pdf

Forsey, M.; Davies, S. \& Walford, G. (2008). The globalization of school choice? Symposium Books. https://doi.org/10.15730/books.70

Flores, C. \& Carrasco, A. (2013). (Des)igualdad de oportunidades para elegir escuela: preferencia, libertad de elección y segregación escolar. Espacio Público.https://www.espaciopublico.cl/wpcontent/uploads/2016/05/8.pdf

García-Huidobro, J. (2010). Para hacer pública la educación pública. En S. Martinic, y G. Elacqua (Eds.), ¿Fin de ciclo? Cambios en la gobernanza del sistema educativo, 81-100. UNESCO-PUC. https://unesdoc.unesco.org/ark:/48223/pf0000190544

Gobierno de Chile. (1990, 10 de Marzo). Ley No 18.962. Ley Orgánica Constitucional de la Educación. Diario Oficial de la República de Chile. https://www.leychile.cl/Navegar?idNorma $=247551$

Gubbins, V. (2013). La Experiencia Subjetiva del proceso de Elección de Establecimiento Educacional en Apoderados de Escuelas Municipales de la Región Metropolitana: a subjective experience. Estudios pedagógicos, 39(2),

165-178.

https://scielo.conicyt.cl/scielo.php?script=sci_arttext\&pid=S071807052013000200011

Hernández, M. \& Raczynski, D. (2015). Elección de escuela en Chile: De las dinámicas de distinción y exclusión a la segregación socioeconómica del sistema escolar. Estudios pedagógicos, 41(2), 127-141. https://scielo.conicyt.cl/pdf/estped/v41n2/art08.pdf

Lomas, C. (2007). ¿La escuela es un infierno? Violencia escolar y construcción cultural de la masculinidad. Revista de Educación, 342, 83-101. http://www.educacionyfp.gob.es/dam/jcr:030aa0e0-fb28-4a73-827973ff79eb4e5f/re342-pdf.pdf

González, J. y Parra, D. (2016). Privatización de la provisión educativa en Chile: Caracterización de los proveedores educativos de una comuna popular urbana. Educação \& Sociedade, 37, 91-112.

López, V.; Carrasco, C.; Morales, M.; Ayala, Á.; López, J. y Karmy, M. (2011). Individualizando la Violencia Escolar: Análisis de Prácticas Discursivas en una Escuela Municipal de la Región de Valparaíso. Psykhe, 20(2), 7-23. http://www.psykhe.cl/index.php/psykhe/article/view/00002

Mizala, A., \& Torche, F. (2012). Bringing the schools back in: the stratification of educational achievement in the Chilean voucher system. International Journal of Educational Development, 32(1), 132-144. http://www.educacion2020.cl/sites/default/files/estudio_mizala_torche.p df

Organización para la Cooperación y el Desarrollo Económicos (OCDE). (2004). Revisión de políticas nacionales de educación. OCDE. https://read.oecdilibrary.org/education/revision-de-politicas-nacionales-de-educacionchile_9789264021020-es\#page14

Organización para la Cooperación y el Desarrollo Económicos (OCDE). (2011). 
Revista de la Escuela de Ciencias de la Educación. 2022, Año 18 1(17), 189 204. Enero a junio. Padilla-Fuentes, G.; Rodríguez-Garcés, C. y Espinosa Valenzuela, D. Segregación y despoblamiento de la matrícula de escuelas públicas en Chile: un estudio de tendencia entre los años 2003 y 2018.

Education at a Glance 2011. OECD Indicators. https://www.oecd.org/education/skills-beyond-school/48631582.pdf

Paredes, R. y Pinto, J. (2009). ¿El fin de la educación pública en Chile? Estudios de economía, 36(1), 47-66. http://econ.uchile.cl/uploads/publicacion/2b0025de-ee94-4c53-8471431ae5804722.pdf

Quijada, A. V. y Mungarro, J. (2014). El proceso de admisión de las escuelas normales y los antecedentes socioeconómicos como predictores del rendimiento académico. Revista Intercontinental de Psicología y Educación, $16(2)$,

111-129. https://www.redalyc.org/pdf/802/80231541007.pdf

Rich, P. \& Jennings, J. (2015). Choice, information, and constrained options: School transfers in a stratified educational system. American Sociological Review, 80(5), 1069-1098. https://doi.org/10.1177/0003122415598764

Santos, H., y Elacqua, G. (2016). Segregación socioeconómica escolar en Chile: elección de la escuela por los padres y un análisis contrafactual teórico. Revista CEPAL, 119 , https://repositorio.cepal.org/handle/11362/40396

133-148.

Tijmes, C. (2012). Violencia y clima escolar en establecimientos educacionales en contextos de alta vulnerabilidad social de Santiago de Chile. Psyhke, 21(2), 105-117. http://www.psykhe.cl/index.php/psykhe/article/view/548

Valenzuela, J. (2008). Evolución de la Segregación Socioeconómica de los Estudiantes Chilenos y su Relación con el Financiamiento Compartido. Proyecto FONIDE $\quad \mathrm{N}^{\circ}$ : $211 \quad$ - 2006. http://www.opech.cl/bibliografico/Doc_Financiamiento/evolucion_segreg acion_chilena.pdf

Van Zanten, A. (2003). Middle-class parents and social mix in French urban schools: reproduction and transformation of class relations in education. International Studies in Sociology of Education, 13(2), 107-124. https://doi.org/10.1080/09620210300200106 